\title{
Aero-Optical and Hot-Wire Measurements of the Flow Around the Hemispherical Turret With a Flat Window.
}

\author{
Stanislav Gordeyev ${ }^{*}$ \\ University of Notre Dame, Notre Dame, IN, 46556 \\ Tim E. Hayden ${ }^{\dagger}$ \\ US Air Force Academy, Colorado Spring, CO, 80840 \\ and \\ Eric J. Jumper \\ University of Notre Dame, Notre Dame, IN, 46556
}

\begin{abstract}
Extensive investigation of the flow over the semispherical turret with the flat window was performed in order to document optical distortions over the window using 2-dimensional wavefront sensor and the Malley probe, complemented with simultaneous Malley probe - single hot-wire measurements of streamwise component velocity's profiles normal to the window at several points across the window's aperture for different azimuthal angles and a range of Mach numbers. The results provide the levels of unsteady optical aberration across the window's aperture, as well as the local thickness, intensity and a convective speed of the separated flow over the window. Results reveal that the optical distortions grow approximately as a square of the incoming Mach number multiplied by a freestream density, $\mathbf{O P D}_{\mathrm{rms}} \sim \rho \mathbf{M}^{2}$.
\end{abstract}

\section{Motivation.}

When an otherwise-collimated laser beam passes through a variable-index-of-refraction turbulent flow its wavefront becomes dynamically (unsteady) aberrated. These aberrations degrade the beam's ability to be focused in the far field, thereby reducing the system utility of the beam that may be used for communication, interrogation and targeting or as a directed-energy weapon. When the laser platform is an aircraft, the two main causes of beam degradation are the thin-layer and immediate air flow around the aircraft, referred to as the aero-optic problem ${ }^{1}$; and the intervening, orders-of-magnitude-longer propagation path through the atmosphere to the target, referred to as the atmospheric-propagation problem. Modern beam-control, adaptive-optic methods appear to now be able to mitigate the atmosphericpropagation effects on the beam; however, both the spatial and temporal bandwidths of the aero-optic problem place it well outside the capabilities of these traditional approaches ${ }^{2}$. It has only been a decade since the first time-resolved wavefront measurements for propagation through a relevant aero-optic flow field were made ${ }^{3}$; prior to that time, aero-optic propagation environments were characterized by limited time-unresolved interferograms and indirectly inferred from hot-wire anemometry techniques ${ }^{1,2}$. In general, the paucity of such characterizations that are available treated the aero-optic problem as a stochastic problem and reduced the measurements to very-unspecific measures of optical degradation as root-meansquare optical path difference, $\mathrm{OPD}_{\mathrm{rms}}$. Such measures, while providing an estimation of the degradation that might be expected, provided little in the way of higher-order information about the aberrating environment's aberration coherence length (spatial bandwidth) and temporal bandwidth over relevant laserbeam apertures. The lack of such characterizations makes it impossible to either infer the far-field degradation in the point spread function or address the requirements for adaptive-optic mitigation schemes.

\footnotetext{
* Assistant Research Professor, Department of Aerospace and Mech. Eng., Member AIAA.

${ }^{\dagger}$ Aerospace Engineer, Aeronautics Department, USAFA, Senior Member AIAA.

* Department of Aerospace and Mech. Eng., Professor, Fellow AIAA

Copyright (c) 2004 by S. Gordeyev, T. Hayden, E. Jumper, Published by the American Institute of Aeronautics and Astronautics, Inc. with permission.
} 
The ability to collect copious spatial and temporal wavefront information through relevant aero-optical type flow fields changed abruptly with the invention by Malley et $\mathrm{al}^{4}$, of a new approach to interrogating these fields with a direct optical method that used a single, small-aperture laser beam at a single location in the larger aperture. Malley at $\mathrm{al}^{4}$ exploited the fact that aberrations due to convecting optically-active turbulence themselves convect through the aperture. This approach was further enhanced with the introduction of multiple beams over the aperture to construct an actual time-resolved one-dimensional wavefront sensor in the flow direction, the small-aperture-beam technique, $\mathrm{SABT}^{5}$. As more experience has been gained investigating aero-optical flow fields, it became apparent that returning to configurations more akin to Malley et. al's original idea of a single interrogation location over an aperture provided an extremely powerful aero-optical measurement tool. One year ago we introduced a new, closely-coupled, two-small-aperture-beam instrument (and accompanying analysis) wavefront-characterization instrument we refer to as a Malley Probe ${ }^{6}$. This instrument represented a new milestone in being able to extract aerooptical and flow information from a single, simple-to-use instrument ${ }^{7}$. In a companion paper $^{8}$, the Malley Probe described in Ref 6 is re-described and a comparison with wavefront data from other instruments is given.

While the methods used to characterize aero-optical flow fields prior to a decade ago lacked higher-order information, such little information that was available provided many instances of propagation through flows around geometries that might be used as relevant aircraft-protruding beam directors [see Ref 9 , for example]. On the other hand, while more-recent, time-resolved wavefront studies available in the open literature are at relevant Mach and Reynolds numbers, they are for fundamental flows like planar free shear layers ${ }^{2,3}$ or canonical attached turbulent boundary layers ${ }^{6}$. This paper changes that trend and presents aero-optical data for a generic beam director similar to earlier geometries found in Ref 9, for example. These data are for three viewing angles; all are at an elevation of $30^{\circ}$, one at a $90^{\circ}$ angle back from the incoming flow, one at $100^{\circ}$ and the last for $110^{\circ}$. The beam director has a flat "window" over the aperture, so that these angles represent a weak separation with reattachment near the leading edge of the aperture; a weakly attached, "nearly separated" flow over the aperture; and separated flow over the aperture, respectively. Unlike the fundamental-flow studies, whose optical data was amenable to straight-forward interpretation, the data in this paper is complicated and, like the data of previous decades, difficult to interpret.

Albeit difficult to understand, the data represents a fairly comprehensive collection of complimentary types of data. As mentioned above, the bulk of the optical data was collected using a Malley Probe; however, a complimentary set of non-time-resolved wavefronts over the full aperture were collected. Also, for the first time, simultaneous hotwire measurements were made with the Malley Probe data. While the overall interpretation of the optical/flow environment still presents an incomplete picture of exactly what is going on, these new Malley-Probe/hotwire data reveal some interesting physics about the role a recirculation region over the aperture plays in the overall optical environment.

\section{Experimental Set-Up.}

All measurements were performed in a closed-loop, subsonic wind tunnel located at the US Air Force Academy in Colorado Springs. The tunnel cross-section is 3 feet by 3 feet with the Mach number range from 0 to 0.6 . The tunnel has three $1 \times 1$ feet optical access windows on the front and back tunnel walls and is equipped with a 3 -dimensional traverse system.

Wind tunnel tests were conducted on the hemisphere-on-cylinder "turret" model shown mounted in the roof of the Academy tunnel in Figure 1. The 12-inch diameter hollow sphere, made of plastic, was re-enforced by adding epoxy material to create a thicker wall. A cylindrical hole was milled in the sphere in order to mount a custom-made flat mirror of high optical quality (less than 1/10 of wave). The round mirror diameter was 5.4 inches. The mirror was held in place by several screws around its perimeter. The slope discontinuity between the sphere and the mirror is 27.5 degrees. The sphere was mounted on a hollow cylindrical base, 12 inches in diameter and 4 inches tall. The sphere can be rotated along a horizontal axis, thus adjusting to any elevation angle between 25 and 90 degrees. The cylinder was mounted on a flat plate and can be rotated to any azimuthal angle. The open cylinder's end was sealed with a flat aluminum plate, so that the interior of the beam director was nominally at the test-section static pressure. 


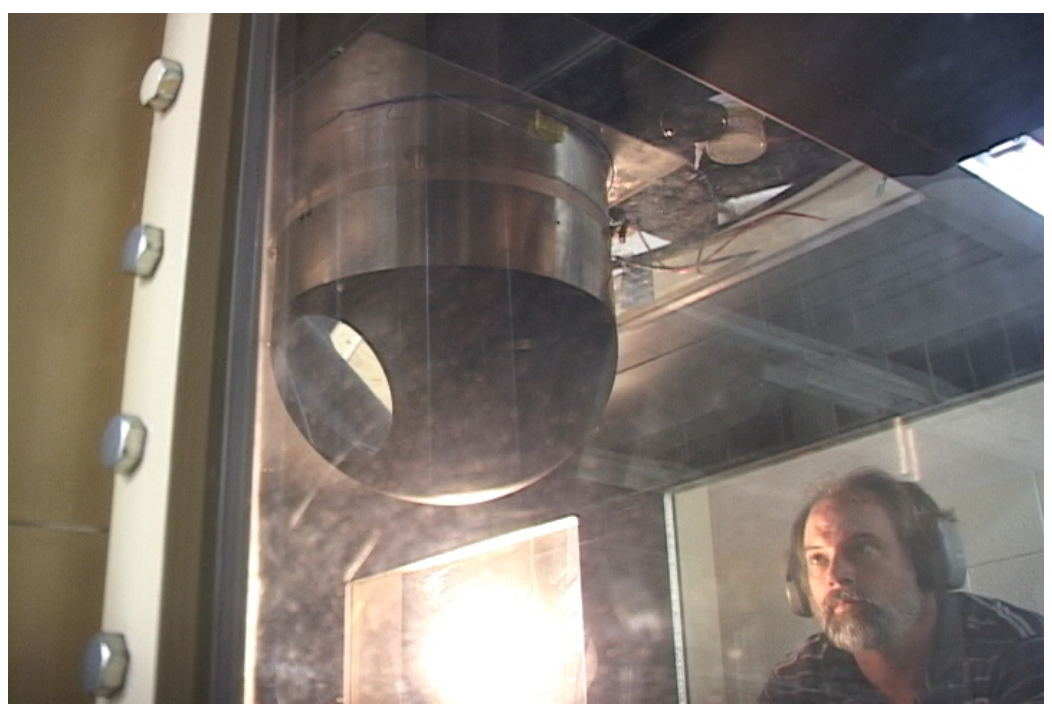

Figure 1. The turret assembly installed in the wind tunnel.

The beam-director's system of coordinates is shown in Figure 2, left. The origin is placed at the center of the flat mirror. The $x$-axis and the $y$-axis lie in the mirror's front surface plane, with the $x$-axis aligned horizontally and the $y$-axis being normal to the $\mathrm{x}$-axis. The $\mathrm{z}$-axis is normal to the mirror surface.

The flow over the turret was studied for one elevation angle of 30 degrees and three azimuthal angles of 90,100 and 110 degrees relative to the flow heading. Thus, the mirror was either faced normal to the incoming flow (90 degrees) or slightly downstream (100 and 110 degrees). Various Mach numbers were studied ranging from $\mathrm{M}=0.3$ to 0.5 . Spatial topology of optical aberrations normal to the mirror's surface were measured by a 2-D, Wavefront Sciences wavefront sensor and extensive studies of optical distortions at several locations on the mirror were performed using the Malley probe. To characterize the flow field, a single hot-wire was used to obtain velocity profiles normal to the mirror at several locations for two azimuthal angles of 100 and 110 degrees. In addition, in order to locate optically-active structures in the normal direction, simultaneous velocity and optical aberrations were conducted in the z-direction at 2 locations over the mirror at a Mach number of 0.37 for one azimuthal angle of 110 degrees using a single hot-wire and the Malley probe.
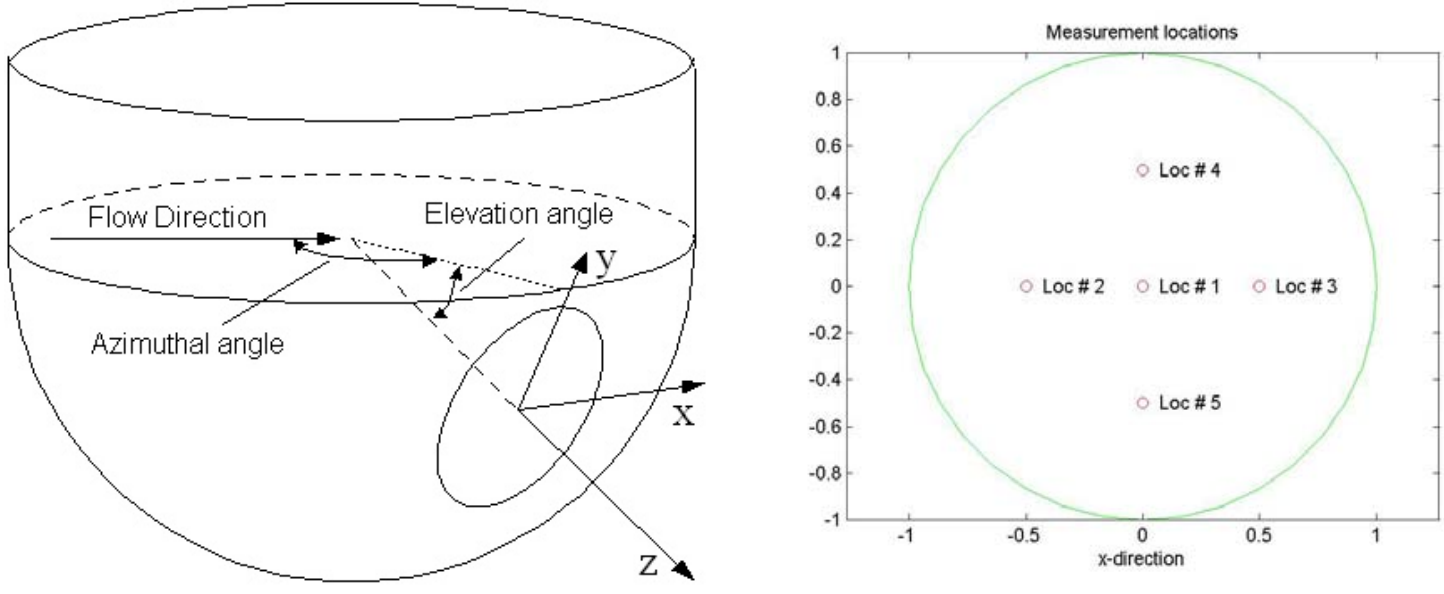

Figure 2. Left: Turret's system of coordinates. Right: Hot-wire and Malley probe measurement locations at the mirror, normalized by the mirror radius. 


\section{Results.}

\section{A. Hot-wire probe}

The velocity profiles in the normal direction were taken for $\mathrm{M}=0.35$ for two azimuthal angles 100 and 110 degrees for Locations \#2, \#1 and \#3, indicated in Figure 2, right. Data were collected at 100 $\mathrm{kHz}$ sampling rate for 10 seconds. Results for 100 degrees are shown in Figure 3 . The flow trips and separates at the leading edge of the mirror, but faces a mild adverse pressure gradient of a 10-degree backfacing mirror, so it re-attaches over the mirror, forming a weak separation bubble at the mirror's leading edge. Thus, the velocity profiles in Figure 3 show the presence of a boundary-layer-like flow over the mirror with growing thickness and relatively low normalized turbulence level from 0.05 to 0.1 which decays downstream.
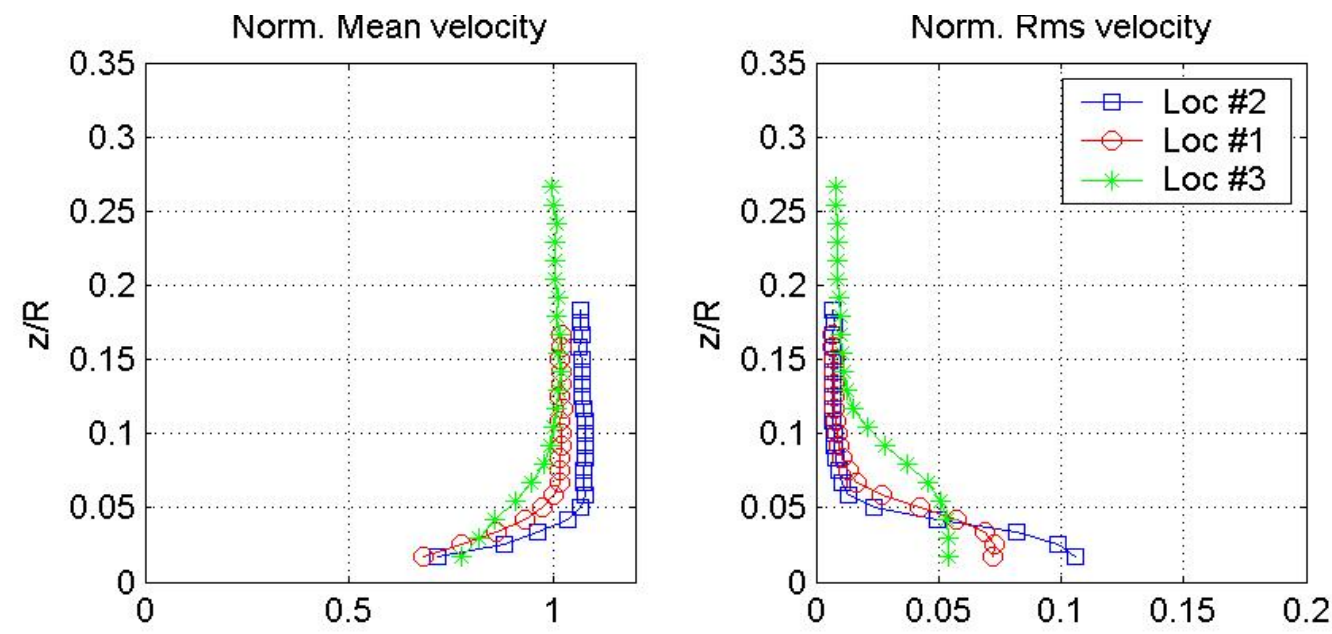

Figure 3. The mean and fluctuating velocity profiles at three locations at the mirror for $\mathbf{M}=\mathbf{0 . 3 5}$ for the azimuthal angle of $\mathbf{1 0 0}$ degrees. Velocities are normalized by the freestream speed.
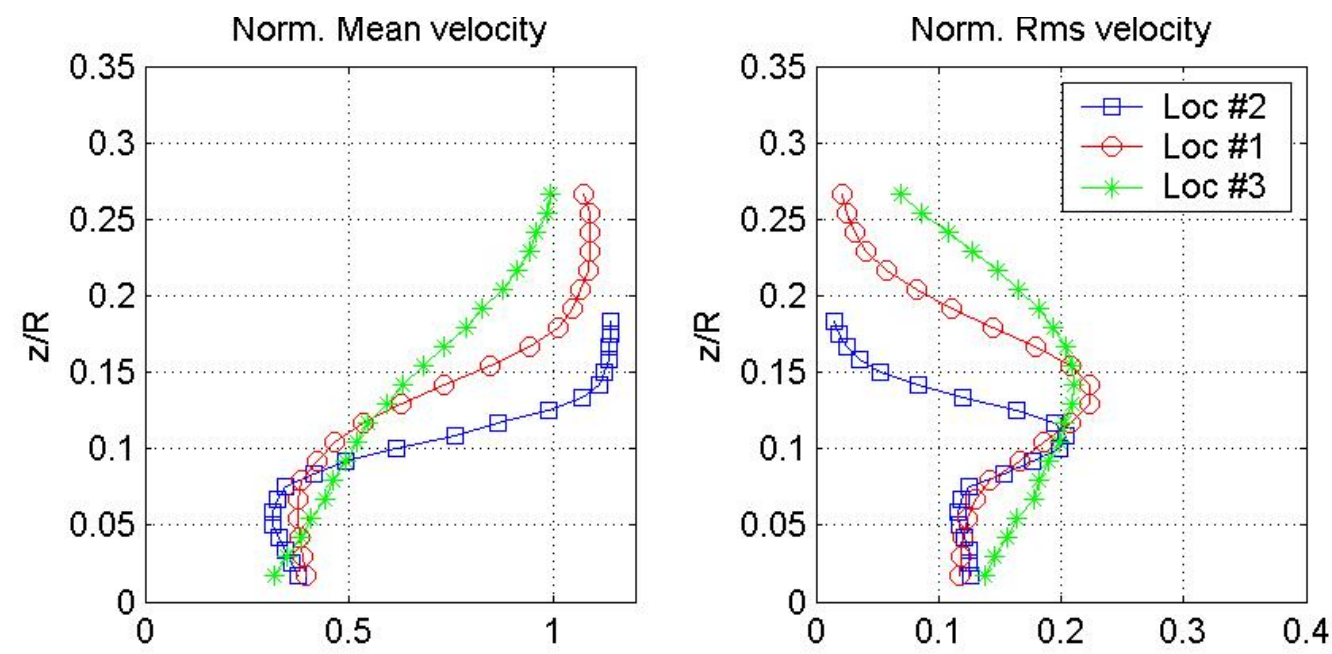

Figure 4. The mean and fluctuating velocity profiles at three locations at the mirror for $\mathbf{M}=\mathbf{0 . 3 5}$ for the azimuthal angle of $\mathbf{1 1 0}$ degrees. Velocities are normalized by the freestream speed.

Results for 110 degrees are presented in Figure 4. At 110 degrees, the flow now faces a stronger adverse pressure gradient over a 20-degree back-facing mirror and clearly remains separated over the mirror. The velocity profiles exhibit a shear-layer-like behavior with a high speed being the free-stream speed and the low speed being around 0.4 of the freestream speed. The shear layer thickness grows 
downstream, indicating the formation of shear-layer, Kelvin-Helmholtz-driven, coherent structures. The turbulence levels have a single peak in the normal direction with maximum turbulence levels of 0.2 of the freestream speed, which is $2-4$ times higher than for the 100 degree case.

\section{B. 2-D wavefronts}

In order to avoid the complications of either injecting or extracting laser beams through the beam director, a more-utilitarian approach was taken for collecting both 2-D wavefronts and the Malley probe data described later. The approach was to use a high-quality optical flat with first-surface mirroring as a surrogate beam-director "window". This approach allowed all the optical components, including the beamsteering mirrors and various laser sources and sensors to share a single optical bench. The optical set-up is shown schematically in Figure 5.

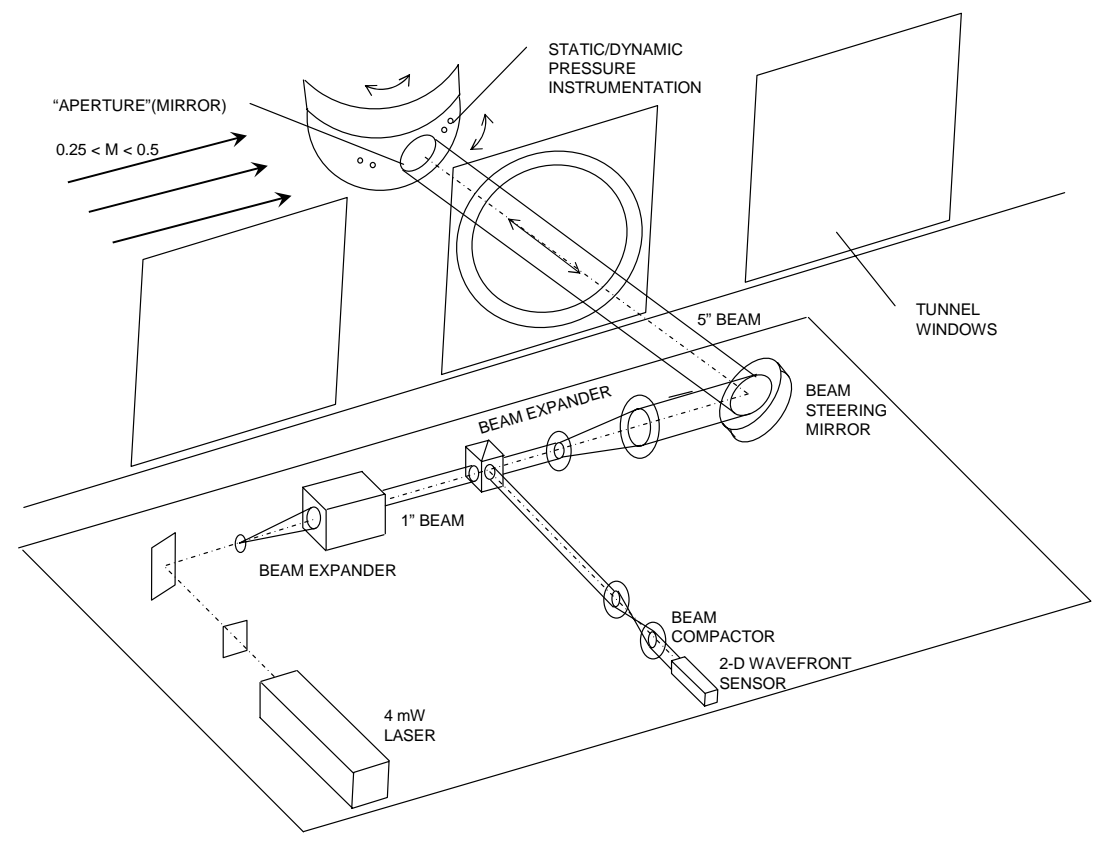

Figure 5. 2-D wavefront experimental set-up.

For the wavefront measurements a $4 \mathrm{~mW} \mathrm{CW} \mathrm{He-Ne} \mathrm{laser} \mathrm{was} \mathrm{expanded} \mathrm{to} \mathrm{a} \mathrm{1-inch} \mathrm{collimated}$ beam, which in turn was expanded to a 4-inch beam. The 4-inch beam was steered into the wind tunnel test section normal to the mirror. The turret mirror reflected the beam coaxially back onto the optical bench, where it was split using a beam-splitter cube. Thus, the beam went through the turbulent flow twice, increasing the signal-to-noise ratio of the aberration measurement. Finally, after a pair of contracting lenses, the beam's optical distortions were measured using a 2-D Hartmann-Shack wavefront sensor with a 33 x 44 lenselet array mounted in front of a CCD camera. The exposure time was 1/10,000 of a second. The wavefronts were sampled at a $30 \mathrm{~Hz}$ sampling rate. Several hundred wavefronts were recorded for each case.

The wavefronts were post-processed as follows: first, each wavefront was constructed as a change from the reference no-flow wavefront to eliminate optical aberrations imposed on the collimated laser beam by the optical set-up; both steady and unsteady tip/tilt and piston modes were then removed from each wavefront. A steady wavefront was computed by averaging all resulting wavefronts in time and then, it was also removed from each wavefront. Thus, the final wavefronts contain only the unsteady tilt-removed aberrations. They reveal the topology of the convective, optically-active structures passing over the aperture.

Two respective wavefronts, treated as above, for 110 degree look back angle are shown in Figure 6 with their concomitant instantaneous component far-field point-spread functions (focus). Although they are single realizations, they are representative of all the wavefronts recorded at these conditions. Their topologies are consistent with the inferences drawn from the hot-wire data, they suggest that opticallyactive elongated shear-layer-like structure form and convect over the aperture. 

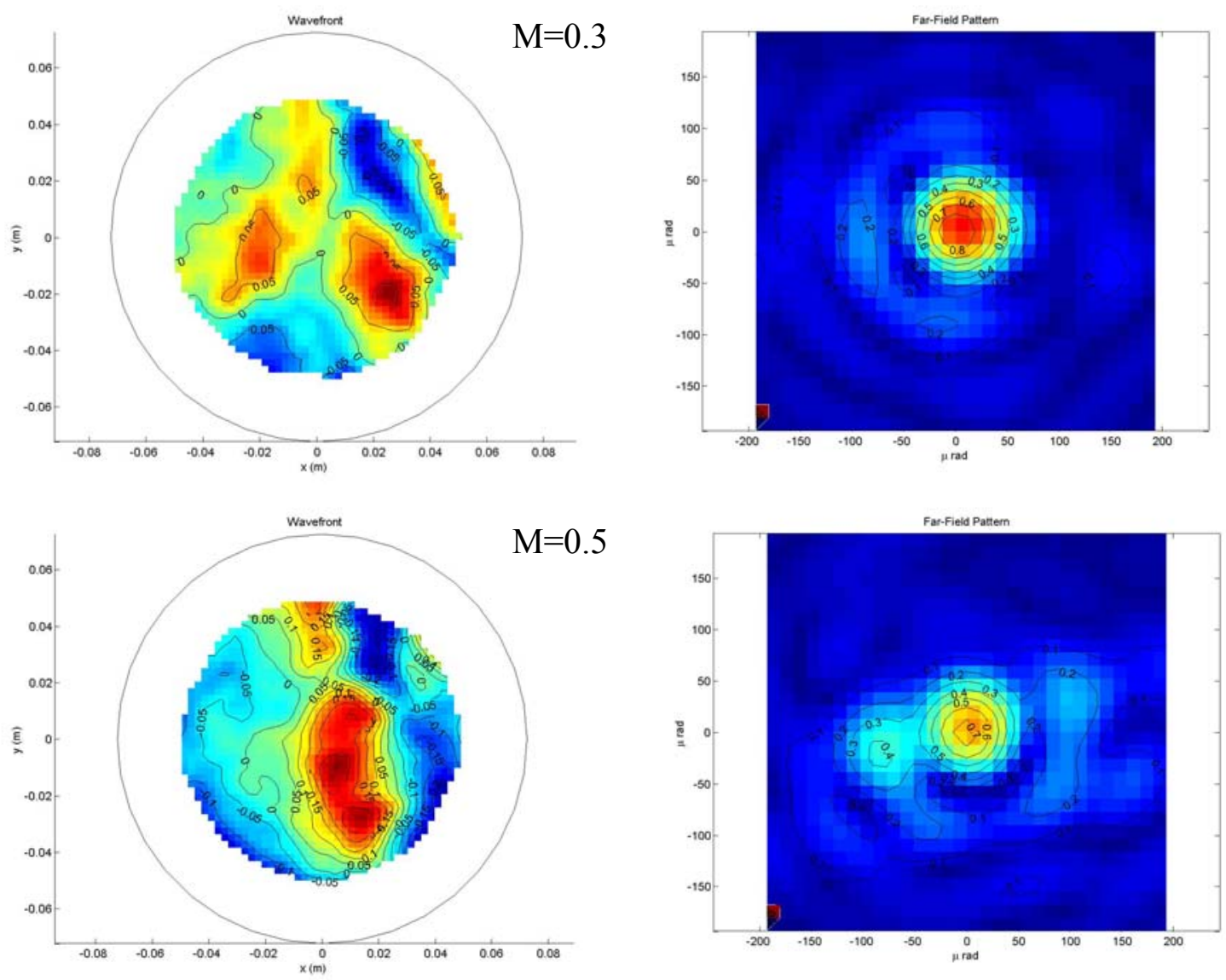

Figure 6. Wavefronts (left) and far-field patterns @ 1micron (right) for $\mathbf{M = 0 . 3}$ and $\mathrm{M=0.5}$. The azimuthal angle is $\mathbf{1 1 0}$ degrees. The mirror is represented by a black circle. The flow goes from left to right.

To characterize the level of optical distortions, the spatial root-mean-square optical path difference over the aperture was calculated for each wavefront, and a time-averaged $\mathrm{OPD}_{\mathrm{rms}}$ was found for each test case. Far-field patterns normalized by the diffraction-limited intensity were also computed for a wavelength of $\lambda=1$ micron.

Table 1. OPD ${ }_{\text {rms }}$ results from 2-D wavefront sensor.

\begin{tabular}{|c|c|c|}
\hline Azimuthal Angle $\theta$ & $\mathrm{M}$ & OPD $_{\text {rms }}$ \\
\hline 90 & 0.3 & 0.0254 \\
\hline 90 & 0.4 & 0.0383 \\
\hline 100 & 0.3 & 0.0577 \\
\hline 100 & 0.4 & 0.0847 \\
\hline 110 & 0.3 & 0.0653 \\
\hline 110 & 0.4 & 0.0856 \\
\hline 110 & 0.5 & 0.0985 \\
\hline
\end{tabular}

Levels of optical aberrations increase with Mach number, resulting in losing the peak intensity in the far-field pattern. Summary of $\mathrm{OPD}_{\mathrm{rms}}$ for all measured cases are presented in Table 1. Clearly the optical aberrations increase with increasing azimuthal angle and Mach number at any particular angle. As indicated in Figure 6 (right), the beam's focus begins to fractionate as the Mach number increases. 


\section{Malley Probe}

To obtain time-resolved unsteady wavefront data, the Malley probe was used to measure optical aberrations at five locations over the aperture (mirror), see Figure 2, right. The detailed description of how these Malley probe data were reduced is given i ${ }^{6,8}$. Data were sampled at $100 \mathrm{kHz}$ rate for 10 seconds. A beam separation of $5 \mathrm{~mm}$ in the streamwise direction was used.

The Malley probe measures the jitter angles of two beams $\delta_{1}(t)$ and $\delta_{2}(t)$ separated in the streamwise direction by a fixed distance $\Delta$. A cross-correlation spectral function $S(f)$ was computed as an ensemble-averaged product of Fourier transformed signals, $S(f)=\left\langle\hat{\delta}_{1}(f) \hat{\delta}_{2}^{*}(f)\right\rangle$.

For stationary disturbances the phase of the S-function $\operatorname{Arg}[S(f)]$ is zero and for pure convective disturbances moving with a convective speed $U_{c}$ the phase of the S-function should grow linearly with frequency, $\operatorname{Arg}[S(f)]=2 \pi \mathrm{f} \Delta / U_{c}$, see Refs 6 and 8. Optical $\operatorname{OPD}\left(t, x_{0}\right)$ can be reconstructed from the jitter signals in the $\mathrm{x}$-direction using the Taylor frozen field hypothesis,

$$
O P L\left(t, x_{0}\right)=-U_{c} \int^{t} \delta_{1}(t) d t, \quad O P D\left(t, x_{0}\right)=O P L\left(t, x_{0}\right)-\overline{O P L\left(t, x_{0}\right)}
$$

Phase plots $\operatorname{Arg}[S(f)]$ versus frequency at the location at the center of the mirror (Location \# 1) for two azimuthal angles of 100 and 110 degrees for $\mathrm{M}=0.4$ are presented in Figure 7 . The phase values hover around zero up to $\sim 1 \mathrm{kHz}$, and then become linearly increasing with frequencies above $1 \mathrm{kHz}$. This indicates that jitter data below $1 \mathrm{kHz}$ is due to stationary effects like vibrations or separation-bubble breathing and are filtered from the data. Above $1 \mathrm{kHz}$, knowing the phase slope, allows for the computation of the mean convective speed of optically active structures.

Since we are interested in convective unsteady structures only, deflection angles were high-pass filtered at $1 \mathrm{kHz}$ to remove any stationary aberrations from signals before computing the convective speeds and OPDs for each case. Results for locations \# 1, 3, 4 and 5 are given in Figure 8. Convective speeds were in a range from 0.6 to 0.8 of the freestream speed $U_{\text {inf. }}$ These values of convective speed are consistent with hot-wire data, presented in Figures 3 and 4. For boundary layers, the convective speed should be 0.8 of the freestream velocity ${ }^{6}$ and for shear layers the convective speed is the half-sum of the speeds above and below the shear layer, thus in this case giving $\sim 0.75$ of the freestream speed.
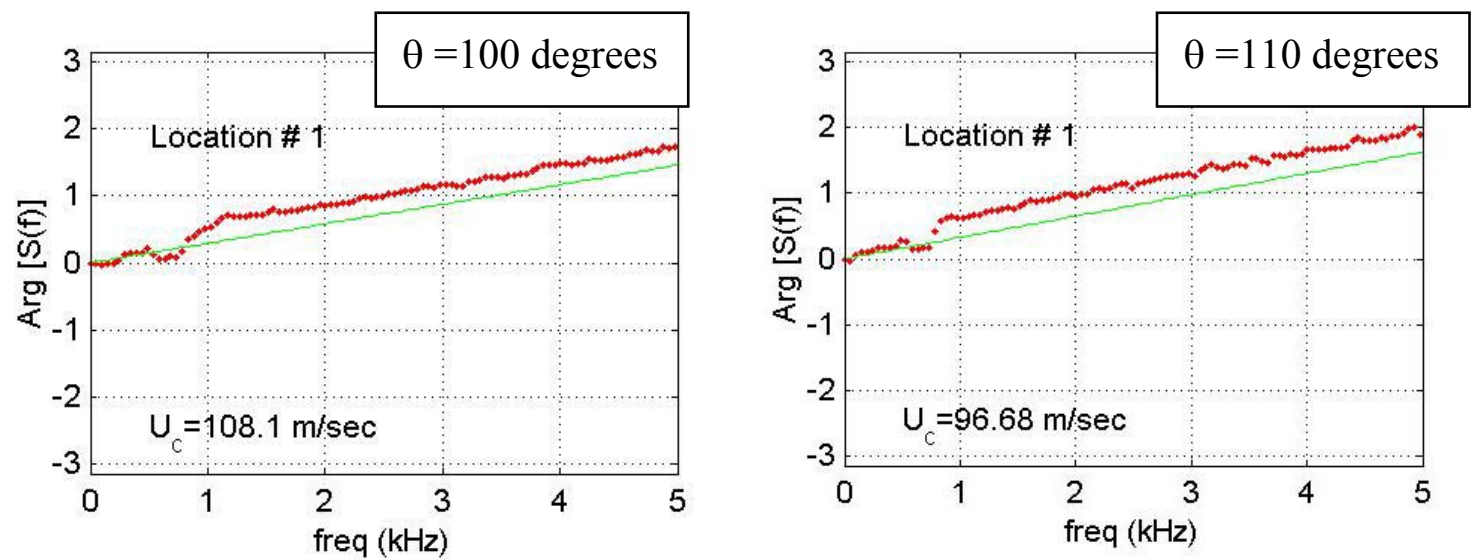

Figure 7. Phase plots Arg [S(f)] for the location \# 1 for the azimuthal angle of 100 (left) and 110 (right) degrees at $\mathrm{M}=0.4$.

Having the $\operatorname{OPD}\left(t, x_{0}\right)$ at each location, time and position can be exchanged using $\mathrm{U}_{\mathrm{c}}, x=-U_{\mathrm{c}} t$, to project the Malley probe data into a pseudo wavefront in the up- and downstream direction as described in Refs 6 and 8. In the present case each location's data was extrapolated to a 4-inch aperture, after which, every realization had local piston and tilt components removed, frame by frame. The subsequent wavefront histories then had spatial room-mean-square OPD computed over the aperture, frame by frame, and the spatial-averaged OPDs were then ensemble-averaged over the entire record. The resulting average aberrations are presented in Figure 8. $\mathrm{OPD}_{\mathrm{rms}}$ are lower for the 90 azimuthal angle case and higher for the 100 and 110 degrees cases, which is consistent with the 2-D wavefront measurements. $\mathrm{OPD}_{\mathrm{rms}}$ grow with 
Mach number as $\mathrm{OPD}_{\mathrm{rms}} \sim \rho \mathrm{M}^{2}$ for most locations, except for the 100 degree case, where the aberrations increase at a rate higher than $\sim \rho \mathrm{M}^{2}$. One possible explanation is that the weak separation bubble over the mirror gets stronger with $\mathrm{M}$, thus increasing the role in the optical aberrations. While the overall aberration level increases with Mach number, consistent with the findings of the 2-D wavefronts given in Table 1, specific locations show individual trends due to the complex interactions of the fluid mechanics over mirror. This is particularly noticeable at location \# 1 for the 110 degree case where the $\mathrm{OPD}_{\mathrm{rms}}$ actually drops at $\mathrm{M}=0.5$. The reason for this behavior is still not fully understood and may be in part due to the role of the breathing separation region, which may occur at less then $1 \mathrm{kHz}$. Overall, the $\mathrm{OPD}_{\mathrm{rms}} \sim \rho \mathrm{M}^{2}$ trend is well-observed for most locations and can be used to scale the results for different Mach numbers and freestream densities.
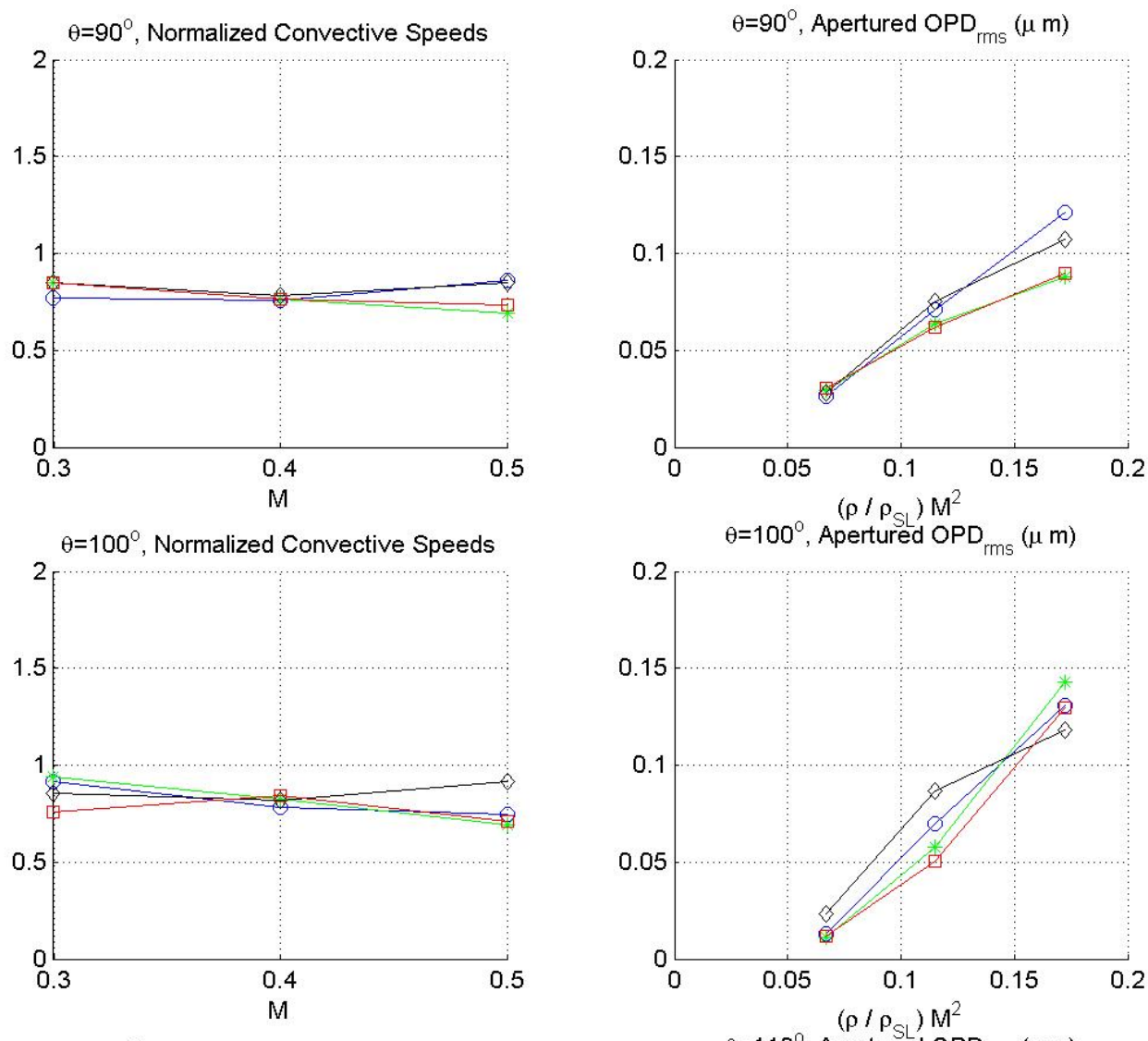

$\theta=110^{\circ}$, Normalized Convective Speeds
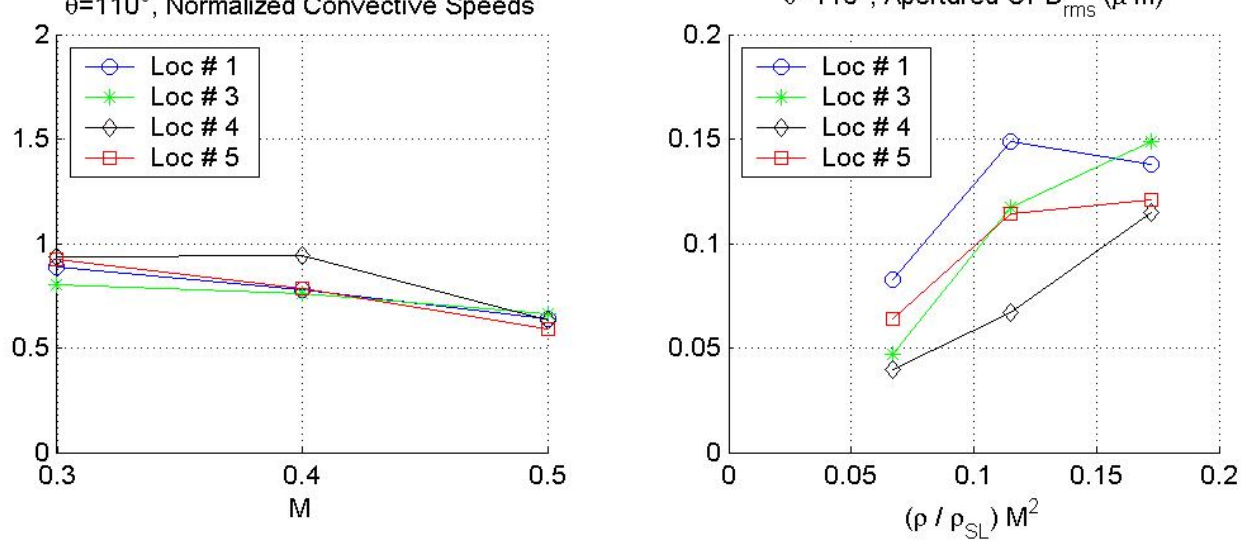

Figure 8. Convective speeds and apertured $O P D_{\text {rms }}$ for different cases.

American Institute of Aeronautics and Astronautics 

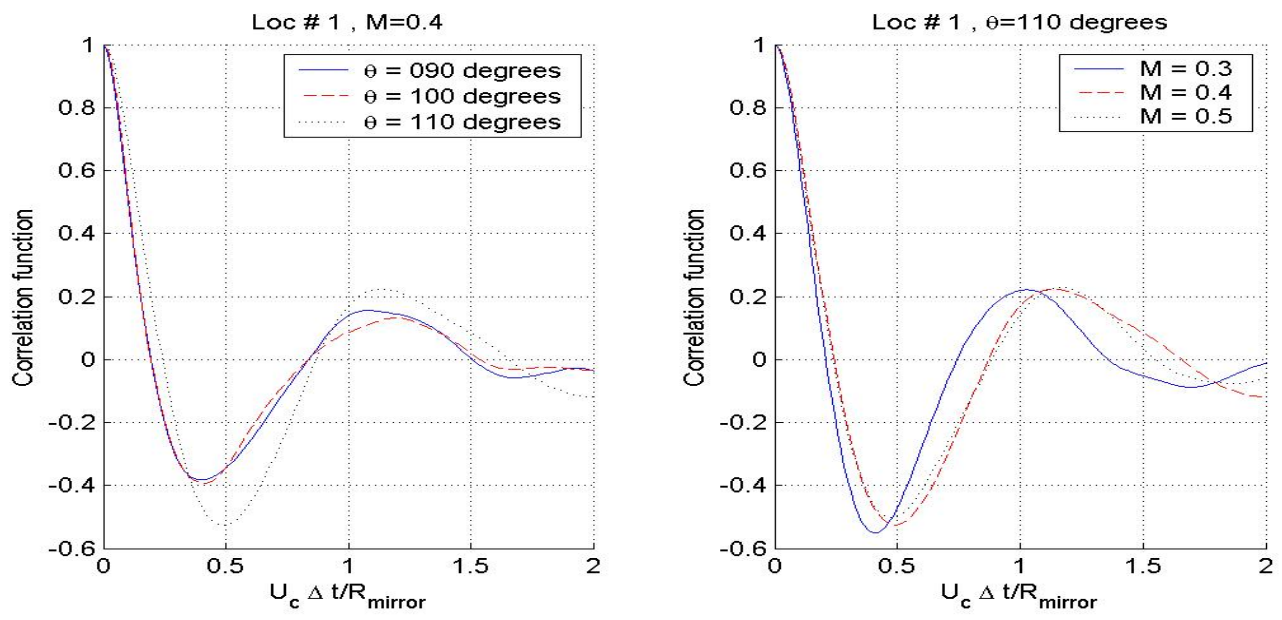

Figure 9. OPD auto-correlation function for the location \# 1.

Streamwise correlation lengths can be obtained from the unapertured Malley probe data by autocorrelating the OPD traces. Results for the middle location \# 1 at the mirror are presented in Figure 9. In the left plot of this Figure Mach number was fixed at $\mathrm{M}=0.4$ and the azimuthal angle was varied. If one uses the definition of the correlation length as location of the first minima, then the correlation length at this location for $M=0.4$ increases from $0.4 R_{\text {mirror }}$ at for $\theta=90$ and 100 degrees to $0.5 R_{\text {mirror }}$ at $\theta=110$ degrees. Similar trends were observed for all other locations. Although the weak growth in the structure is present, there is an uncanny similarity in not only the one structure but in the overall decay in correlation with subsequent structures. This is an important point because while we have noted that at 90 and 100 degrees the flow measurements indicate a boundary-layer like flow, in fact, the role of the initial separation bubble must be significant. This bubble must be instrumental in forming cross-stream structures which subsequently convect with the turbulent re-attached boundary layer. This "memory" of the separation region in the re-attached boundary layer has been noticed in other flows ${ }^{10}$.
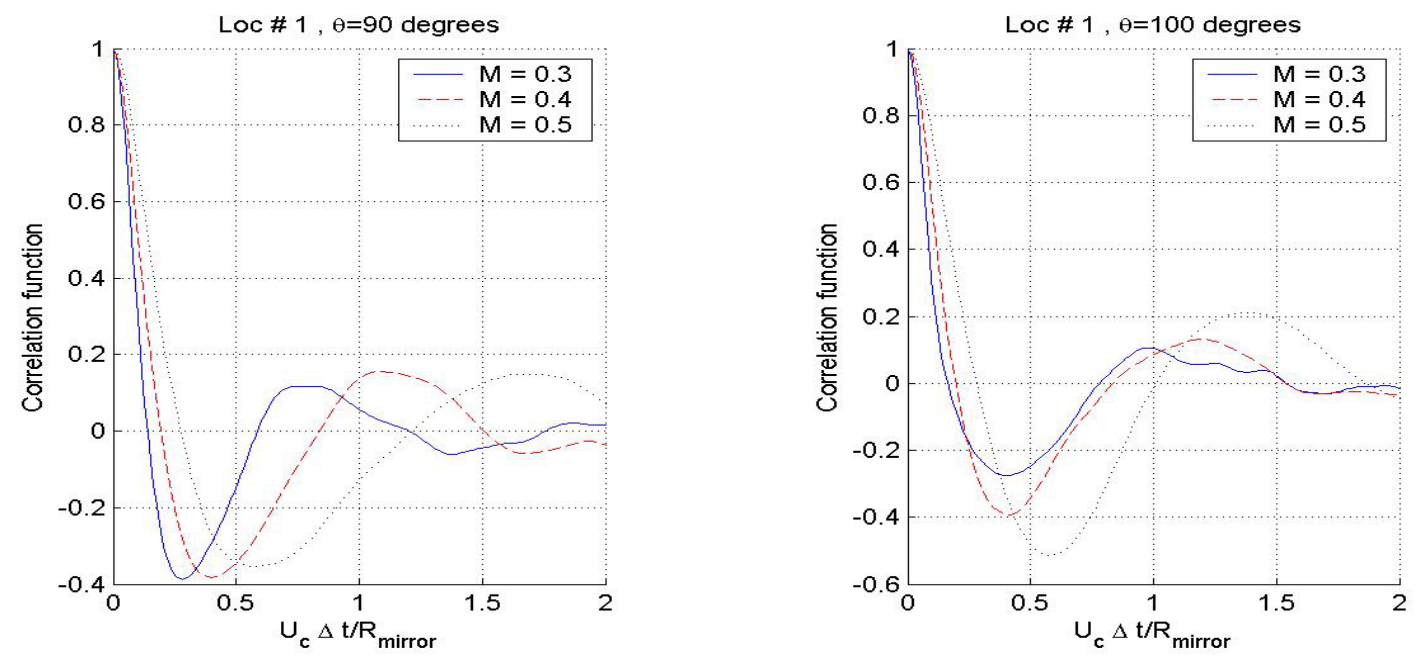

Figure 9. OPD auto-correlation function for the location \# 1 for 90 and 100 degree cases.

In the right plot in Figure 9, auto-correlation functions are plotted for different Mach numbers for the fixed azimuthal angle $\theta=110$ degrees. The correlation length is $0.4 \mathrm{R}_{\text {mirror }}$ for $\mathrm{M}=0.3$ and 0.4 , then slightly increases to $0.5 \mathrm{R}_{\text {mirror }}$ for $\mathrm{M}=0.5$. Inspection of the 2-D wavefronts in Figure 6 gives the similar structure sizes in the streamwise direction. 
A relative independence of correlation length with Mach numbers at 110 degrees indicates a strong separation bubble, which dynamics is independent of the incoming Mach number. But in the case of the weak separation at 90 and 100 degrees the separation bubble is sensitive to the incoming Mach number and the correlation length should vary with M. Indeed, it is the case as it can be seen in Figure 10. Correlation length increases with the incoming $M$ for both 90 and 100 degree cases.

\section{Hot-wire - Malley probe correlations}

Finally, the simultaneous measurements of the local velocity and the Malley probe were performed in an attempt to better understand the role that structures in the various regions at the flow have on the optical aberrations. These simultaneous Malley-probe - single-hot-wire measurements were performed at locations \#1 and \#3 over the mirror, for the azimuthal angle of 110 degrees for one Mach number, $\mathrm{M}=0.37$. Note that the 110 degree case is fully separated flow over the mirror, as discussed in the previous section. The Malley probe beams were spaced horizontally $5 \mathrm{~mm}$ apart and the single hot-wire was traversed in the normal direction $3 \mathrm{~mm}$ above of the downstream beam. Sampling rate was $100 \mathrm{kHz}$ with a sampling time of 1 second.

A zero-time lag cross-correlation function between the velocity signal $\mathrm{u}(z, t)$ and the downstream beam deflection $\delta_{2}(t)$ normalized by a maximum rms of the velocity and the deflection signal was computed,

$$
\operatorname{Corr}(z)=\frac{\overline{u(z, t) \delta_{2}(t)}}{u_{r m s}^{\max }(z) \delta_{2 r m s}}
$$

Results for both locations are shown in Figure 11. At the mirror center location \# 1, the correlation function exhibits relatively high positive values of $\sim 0.25$ on the low speed side of the shear layer (i.e. closer to the mirror) for $\mathrm{z} / \mathrm{R}$ between 0.05 and 0.2 with the peak correlation-function value of 0.3 at the maximum $\mathrm{u}_{\mathrm{rms}}$ location. Further away from the mirror the correlation function quickly decays but retaining small negative values. These negative values are due to Biot-Savart induced unsteady potential fluctuations in the freestream velocity field from the circulation density in the separated shear layer.

Further downstream, at the location \# 3, the correlation function has smaller values of $\sim 0.2$ and has a more localized peak centered at the middle of the shear layer $\mathrm{z} / \mathrm{R} \sim 0.15$. Lower correlation values may indicate a more turbulent or defused-vorticity flow with less crisp optically-aberrating structures.

The overall, non-zero correlation values indicate an important observation, the optically active region over the flat window is located on the low speed side and the middle part of the shear layer.
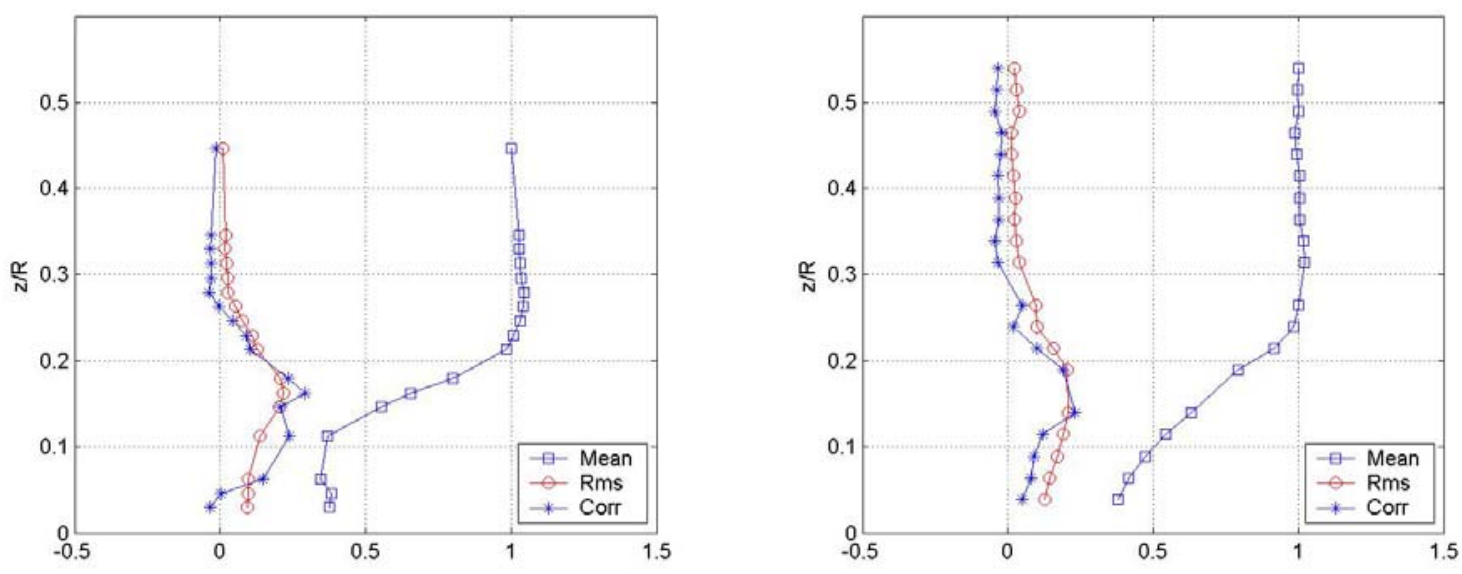

Figure 11. Normalized velocity profiles and the correlation function for the location \# 1 (left) and for the location \# 3 (right). Azimuthal angle is 110 degrees, freestream $\mathbf{M}=0.37$. 


\section{Discussion and Conclusions.}

This paper presented an extensive initial effort to characterize levels of unsteady optical aberrations imposed in an outgoing, collimated laser beam from "hemisphere-on-cylinder" turret arrangement with a flat window. Measurements at several azimuthal angles over a range of subsonic Mach numbers, at a fixed elevation angle, were performed. The optical measurements included both 2-D wavefronts and 1-D Malley probe data. Hot-wire measurements documented the velocity profiles in the normal direction from the flat window and have shown that the flow is separated over the flat window for the azimuthal angles bigger that 100 degrees. Simultaneous hot-wire - Malley probe results reveal that the most optically active region in the separated flow over the flat window is in the shear layer region, biased toward its low-speed side. This means that optical distortions over a flat window are governed mostly by the separated shear layer in an adverse-pressure gradient environment, rather that the unsteady separation bubble formed behind the hemisphere. Thus, a variety of flow control devices which do improve optical aberrations in the separated turbulent flow over the back-facing 2-D ramp ${ }^{10}$, might also work on the turret.

Both 2-D wavefront measurements and properly apertured Malley probe results have revealed that the optical distortions get worse with increasing the azimuthal angle. Also they grow with the incoming Mach numbers and the freestream density as $\rho \mathrm{M}^{2}$. A steady lensing component and an unsteady tip/tilt were removed in the post-processing analysis from both the 2-D wavefront data and the Malley probe results, although the unsteady tip/tilt was removed in a slightly different manner in the two measurements. In the 2-D wavefront data, the actual tip/tilt was removed from each wavefront. In the Malley probe, unsteady tip/tilt was recognized as frequencies with a zero correlation phase between the two Malley probe beams and these frequencies were removed from the jitter signals, prior to computing the OPL. Differences in removing the unsteady tip/tilt might explain a small difference in the $\mathrm{OPD}_{\mathrm{rms}}$ results obtained using the 2-D wavefront and the Malley probe results.

\section{Acknowledgments.}

These efforts were sponsored by the Air Force Office of Scientific Research, Air Force Material Command, USAF, under Grant Number F49620-03-1-0019. The U.S. Government is authorized to reproduce and distribute reprints for governmental purposes notwithstanding any copyright notation thereon.

\section{References}

${ }^{1}$ K. J. Gilbert and L. J. Otten (eds), Aero-Optical Phenomena, Progress in Astronautics and Aeronautics, Vol. 80, AIAA, New York, 1982.

${ }^{2}$ Jumper, E.J., and E.J. Fitzgerald, "Recent Advances in Aero-Optics", Progress in Aerospace Sciences, 37, 2001, pp.299-339.

${ }^{3}$ Hugo, R.J., Jumper, E.J., Havener, G., Stepanek, C., "Time-Resolved Wavefront measurements Through a Compressible Free Shear Layer," AIAA Journal, 35(4), 1997, pp. 671-677.

${ }^{4}$ Malley, M., Sutton, G.W., and Kincheloe, N., "Beam-Jitter Measurements for Turbulent Aero-Optical Path Differences, Applied Optics, Vol 31, 1992, pp. 4440-4443.

${ }^{5}$ Jumper, E.J., and Hugo, R.J., "Quantification of Aero-Optical Phase Distortion Using the Small-Aperture Beam Technique,” AIAA Journal, 33(11), 1995, pp. 2151-2157

${ }^{6}$ S. Gordeyev, E. Jumper, T. Ng and A. Cain, “Aero-Optical Characteristics of Compressible, Subsonic Turbulent Boundary Layers”, AIAA Paper 2003-3606, Orlando, Florida, June, 2003.

${ }^{7}$ For further information contact: Alan B. Cain, ITAC, PO Box 6971, Chesterfield, MO 63006, Ph: 314.576.1639 Fax: 314.576.5620.

${ }^{8}$ D. Duffin, S. Gordeyev and E. Jumper," Comparison of Wavefront Measurement Techniques on a TwoDimensional Heated Jet", AIAA-2004-2446, Portland, Oregon, June, 2004.

${ }^{9}$ J.E. Craig and J.D. Trolinger, "Propagation Diagnostic Technique for Turbulent Transonic Flow", AIAA 840104, Jan, 1984.

${ }^{10}$ S. Gordeyev, E. Jumper, T. Ng and A. Cain, "Optical Disturbances caused by Transonic Separated Boundary Layer Behind a 20-degree Ramp: Physics and Control”, AIAA Paper 2004-0472, Reno, Nevada, January, 2004 